\title{
Energy Aware Knowledge Extraction from Petri Nets Supporting Decision-making in Service- oriented Automation
}

\author{
Paulo Leitão ${ }^{1,2}$, Joel Alves ${ }^{1}$, J. Marco Mendes $^{3}$, Armando W. Colombo ${ }^{4}$ \\ ${ }^{1}$ Polytechnic Institute of Bragança, Campus $\mathrm{S}^{\text {ta }}$ Apolónia, Apartado 1134, 5301-857 Bragança, Portugal \\ \{pleitao, gei10196\}@ipb.pt \\ 2 Artificial Intelligence and Computer Science Laboratory, R. Campo Alegre 102, 4169-007 Porto, Portugal \\ ${ }^{3}$ Faculty of Engineering - University of Porto, Rua Dr. Roberto Frias s/n, 4200-465 Porto, Portugal \\ marco.mendes@fe.up.pt \\ ${ }^{4}$ Schneider Electric Automation GmbH, Steinheimer Str. 117, D-63500 Seligenstadt, Germany \\ armando.colombo@de.schneider-electric.com
}

\begin{abstract}
This paper introduces an approach to decision support systems in service-oriented automation control systems, which considers the knowledge extracted from the Petri nets models used to describe and execute the process behavior. Such solution optimizes the decision-making taking into account multi-criteria, namely productive parameters and also energy parameters. In fact, being manufacturing processes typically energy-intensive, this allows contributing for a clean and saving environment (i.e. a better and efficient use of energy). The preliminary experimental results, using a real laboratorial case study, demonstrate the applicability of the knowledge extracted from the Petri nets models to support real-time decision-making systems in service-oriented automation systems, considering some energy efficiency criteria.
\end{abstract}

\section{INTRODUCTION}

The use of Service-oriented Computing (SOC) and Service-oriented Architectures (SoA) in industrial automation domain seems a suitable and promising computing framework [1]. A primary goal of SOC is to make a collection of software services accessible via standardized protocols, whose functionality can be automatically discovered and integrated into applications or composed to form more complex services [2]. In such systems, the manufacturing processes can be decomposed into smaller equipment pieces, so that each piece of the equipment is abstracted through services. The system behavior is translated into the correct use of these exposed services, as well as the creation of newer ones, in a form of orchestration, composition and association. This way of engineering has been seen to promote the flexibility and reconfiguration capabilities of manufacturing processes.

The logic of the inter-service management can be done using high-level programming, such as Business Process Execution Language (BPEL), Web Services Choreography Description Language (WS-CDL) and Petri nets. The last one is being adopted by the authors mainly due to its mathematical foundation that provide innumerous features, not only in terms of modeling of a service-oriented system, but also in terms of its analysis, validation, simulation and execution [3].

In manufacturing control processes, e.g. those based on service-oriented principles, decision-support systems are crucial to solve conflicts (e.g. which resource should perform the operation or which path should be taken by the pallet) and to recover from unexpected disturbances (e.g. a robot collision during its movement). These conflicts usually require external support by providing a concrete answer to solve the conflict, taking into consideration a set of criteria, such as productivity and efficiency.

The decision support system proposed in this work considers a multi-criteria function that uses the knowledge extracted from the structure of the Petri nets models, used to describe and execute the service-oriented process behaviors. Petri net models contain richness knowledge about the process behavior, namely the description of service and device logics, and available system's work cycles, besides some flexibility in terms of indirections and exceptions that are fundamental for decision systems.

Since nowadays, the issues related to energy efficiency are in the order of the day, an important criterion to be used by such decision support systems is the minimization of energy consumption. In fact, manufacturing is traditionally an energy-intensive industry, using motors, steam, and compressed air systems to transform raw materials into durable goods and consumer products. Adopting more energy-efficient technologies and applying best practices in the control of manufacturing processes, the energy consumed in the factory plant can be significantly reduced and consequently improve the energy efficiency, reduce $\mathrm{CO}_{2}$ emissions and enhance the fuel flexibility. 
Motivated by the importance of energy-aware topics in manufacturing systems, the proposed innovative decisionmaking system supports the conflict resolution in serviceoriented systems, aiming to optimize the manufacturing processes, but also reduce the energy consumption in the manufacturing devices. In terms of services, it means the selection of the best available service from a set of options that represents the requester's demands (in this case, in terms of process and energy efficiency).

The rest of the paper is organized as follows: Section 2 overviews the use of the Petri nets formalism in the description and execution of service-oriented automation systems. Section 3 introduces the proposed approach for the decision support systems considering a multi-criteria function that uses the knowledge extracted from the Petri nets models. Section 4 discusses several criteria that can be applied to perform the decision-making, taking a special attention to the energy related parameters. Section 5 uses a case study to illustrate the applicability of the proposed approach and at last, Section 6 rounds up the paper with the conclusions.

\section{PETRI NETS THEORY BEHIND THE ENGINEERING METHODS FOR THE EXTRACTION OF KNOWLEDGE}

Service-oriented principles are being used to establish a new class of modular and reconfigurable production systems, which considers High-level Petri nets as formal language to describe and execute the structure and predictive behavior of a production system (see [3] for information about the type of High-level Petri nets used). It uses a bottom-up approach, which consists in:

- Modeling the behavior of resources, such as robots, machines and conveyors, using High-level Petri nets. The models represent all possible discrete states of such a resource and also all manufacturing functions that this resource is able to expose as services, e.g. move-piece, pick-part and transfer-pallet. The modeling approach generates a set of resource's discrete states that fulfill basic properties, such as boundedness and conservativeness [4], and a set of resource's exposed services that fulfill basic properties, such as repetitiveness and liveness.

- The resources models are composed into a coordination model. This task follows the same rules of configuring a required resource's layout, i.e. taking into account, amongst others, the competition, concurrency and shared resources behavioral relationships. The result is a High-Level Petri nets model of the whole factory.

Due to the strong mathematical background that is behind the Petri net theory, the models can formally be analyzed and validated [5-7]. Basically, two types of analysis can be made: the qualitative and the quantitative analysis.

The qualitative analysis, based on the structural analysis of the matrix representation of the graph model, allows the verification of the structural and behavioral properties of the High-level Petri nets model, extracting conclusions about the operation of the system, such as the existence of conflicts and deadlocks, the bounded capacity of resources, and the possible control sequences [5]. Place and transition invariants allow verifying mutual exclusion relationships among functions and resources (in case of P-invariants), and the identification of work cycles, i.e. the alternative paths to evolve (in case of T-invariants).

The quantitative analysis can be performed by means of the simulation of the temporized High-level Petri nets models, allowing the verification of the system compliance with specified performance indexes, such as throughput and resource utilization, and the development of optimization strategies.

The knowledge extracted from the High-level Petri nets models, especially the $\mathrm{P}$ - and $\mathrm{T}$-invariants, have a proper meaning in the physical system:

- Co-related discrete states of composed resources belonging to, at least, one state-invariant (P-invariant in the Petri nets terminology). The set of state-invariants and their linear compositions represents all possible configurations of resources that are able to expose a service.

- Co-related exposed services belonging to, at least, one service-invariant ( $\mathrm{T}$-invariant in the Petri nets terminology). The set of service-invariants and their linear compositions represents all possible service coordination paths that the system is able to expose.

The coordination model structure represents the kernel of a service-oriented component identified here as model-based dynamic orchestration engine. When the model-based orchestration engine is interacting with the resources, in realtime production conditions, many well-known intelligent supervisory control and automation functions, like modelbased monitoring, diagnosis and maintenance control, are intrinsically supported and performed. In such systems, the interaction is based on the exchange of events and exposing/calling services.

Note that the application of Petri nets to perform monitoring of flexible production systems has been addressed, among others by [8-9]. One of the major references to model-based monitoring methods and systems is [10] (and references therein):

- The intrinsically contained information about the resource's states, exposed services and controlled manufacturing process, which is contained in the Highlevel Petri nets model, can be recognized as modelbased monitoring indexes.

- The model-based monitoring indexes can also be exposed as services (i.e. model-based monitoring services).

- The model-based monitoring services will be used to enhance the feature-based monitoring indexes, e.g. sensor signals and device-parameters like motorvelocity and electrical intensity, that are exposed as services by the devices. 
Having the minimal set of state- and service-invariants, the dynamic High-level Petri nets orchestration will consist in generating and exposing different compositions of those invariants:

- Each composition is a coordination-path, allowed by the modeled system.

- Each composition will be done by weighting the individual atomic service. It is basically a composition of services-invariants done according to pre-conditions required to automate the behavior of the system, i.e. minimal energy-consumption service-path, faster throughput service-path, etc.

Since the system (e.g. resources and factory) and their High-level Petri nets models are algebraic vectorial fields, the service coordination is respecting all the rules and laws that such vectorial spaces are having [11-12], namely:

- There is an isomorphism between the composition of service invariants coming from the processing/analysis of the High-level Petri nets model (see [13-14]) and the composition of services exposed by the resources/factory.

- The cardinality of the state and service spaces of the real production environment is the same of the places and transitions invariants of the High-level Petri nets model.

Concluding, the knowledge extracted from the High-level Petri nets models, representing the behavior of the physical system, are then useful to be used by decision support systems in service-oriented automation systems, as illustrated in next sections.

\section{DECISION-MAKING BASED ON THE KNOWLEDGE EXTRACTED FROM High-LEVEL PETRI NeTS}

In flexible manufacturing environments it is normal the existence of conflict situations, since there are several alternatives to execute similar operations. Examples of conflicts are the selection of which workstation should perform a drill or which path should be taken to reach a specific workstation. These situations should be handled by decision support systems that will provide services to support the evolution of the system. The complexity of the decision support system is strongly dependent on the flexibility that the system reveals [15].

Since High-level Petri nets are used to describe and execute the behavioral models in the service-oriented automation approach, the knowledge extracted from those models will support the decision system. For this purpose, the following information is considered as basis for the decision process:

- The workplan and the operation to be executed.

- The process behavior and the structure of the model, i.e. the incidence matrix in the Petri nets terminology.

- The current state of the system, i.e. the marking of the net.

- The several sequences of operations, i.e. the Tinvariants in the Petri nets; they can be determined, for example, applying the algorithm described in [16].
- The information of each resource related to several parameters, e.g. efficiency and energy consumption.

Using this set of knowledge, the decision-making system considers two steps: i) determining the allowed possibilities to evolve, and ii) deciding the best alternative to evolve.

\section{A. Determination of Valid Alternatives}

When a decision node is detected, e.g. a conflict, which is represented in Petri nets by places that have multiple output arcs going to different transitions, the decision system should determine, in a first instance, which alternatives are valid to evolve, aiming to reach a particular objective.

Initially, the current marking of the Petri net is crossed with the structure of the Petri net, provided by the incidence matrix, to determine the several options to evolve.

Next, the achieved information, i.e. the several transitions that can fire, is matched with the set of T-invariants to determinate the set of valid alternatives to execute the required service. For this purpose, each T-invariant is checked to find the existence of both the conflict's transition and the transition that represents the service to be executed. The simultaneously existence of both transitions indicates that it is a valid transition to evolve.

As illustrated in Fig.1, and considering that the objective is to perform a drill operation that can only be executed by the transition $t_{4}$, the application of the described method will determine that only the transition $t_{2}$ is a valid alternative to reach the objective.

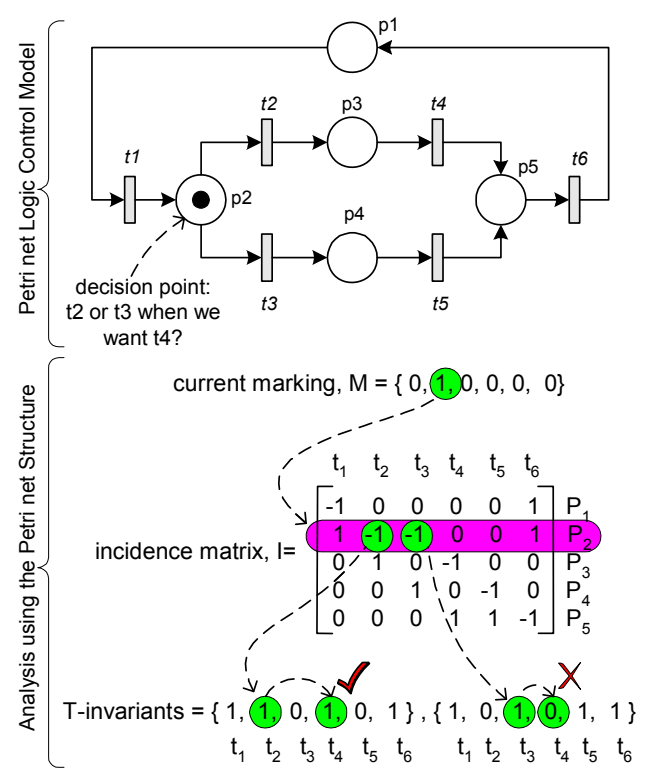

Fig. 1. Determining the valid alternative solutions by using the knowledge extracted from the Petri nets structure [15].

At this stage, the problem is solved if only one alternative is valid to execute the service. However, usually, more than one alternative can be possible. As an example, consider that the objective is to perform the same drill operation, but now it can be executed by transition $t_{4}$ and $t_{5}$. In this case, transitions $t_{2}$ and $t_{3}$ are valid alternatives to achieve the objective. 


\section{B. Evaluation Algorithm}

In case of more than one alternative to evolve it is necessary to evaluate them through an evaluation function that may take into consideration several criteria. It is important to note that reducing the number of alternative sequences (i.e. the T-invariants) means less computer memory and faster processing analysis of the best solution.

The proposed approach for the decision function uses linear algebra operations to combine $\mathrm{T}$-invariants with a vector of decision criteria, where different types of decision parameters can be considered, including productive and energy efficiency related parameters. The set of decision criteria, and particularly the weight of each one, is represented by the vector $w=\left(w_{1}, w_{2}, \ldots, w_{\mathrm{c}}\right)$, being $c$ the number of criteria used in the decision set.

In this method, the alternative solutions are represented by a sub-set of T-invariants, $x=\left(x_{1}, x_{2}, \ldots, x_{\mathrm{m}}\right)$, extracted from the Petri nets model, being $m$ the number of T-invariants. Each T-invariant of the Petri nets model is a vector comprising $\mathrm{n}$ transitions, i.e. $x_{\mathrm{i}}=\left(t i_{1}, t i_{2}, \ldots, t i_{\mathrm{n}}\right)$, for $i=1, \ldots$, $m$. A transition may represent a logic condition or a service (i.e. a time-consuming operation). Each transition representing a service has associated a vector, $t_{i j}=\left(v_{i 1}^{j}, v_{i 2}^{j}, \ldots, v_{i c}^{j}\right)$, for $j=1, \ldots, n$, where $v$ is the performance of the service related to a specific defined criterion, e.g. the processing time and the energy consumption. The values of each criterion for each transition may be constantly updated according to the application particularities and specifications. Note that in case of transitions representing logic conditions, these values are null.

The multi-criteria decision function that evaluates a specific sequence of transitions (given by alternative $\mathrm{T}$ invariants) to execute the next service is then given by:

$$
f(i)=\sum_{j=1}^{n} w t_{i j}^{T}
$$

The value $f(i)$ represents the evaluation score of the sequence path represented by the $i$ T-invariant, considering the set of criteria defined initially and the weight of each criterion. Note that $w t_{i j}^{T}$ is the performance of each transition, included in the $i \mathrm{~T}$-invariant, for the defined set of criteria.

The scores $f(i)$ achieved for each T-invariant $x_{\mathrm{i}}$ can be ranked and the selection of the best solution is easily performed by choosing the solution with a minimal evaluation score when the objective is to minimize the function or to choose the higher score when the objective is to maximize the function.

An important issue to be considered when dealing with a multi-criteria function is related to normalize the dimensions of the different criteria, allowing to compare different things. For this purpose, all the several parameters $v_{i k}^{j}$ associated to the transition are normalized into the interval $[0,1]$.

\section{DECISION CRITERIA FOR ENERGY AWARE MANUFACTURING SYSTEMS}

The evaluation of alternative solutions uses a multi-criteria function. Traditionally, decision-making systems consider productive related parameters as the unique criteria, namely:

- Cost, i.e. the cost to execute the operation by a device.

- Processing time, i.e. the time necessary to execute the operation.

- Location, i.e. the distance to the local where the device that will perform the next operation is placed.

- Resource utilization, i.e. the percentage of utilization of a device.

- Confidence degree, i.e. the level of trust that the device had granted during its historic operation.

- Quality of service, i.e. the quality with which the operation is performed.

- Maintenance, i.e. the cost related with repair and lubrication operations.

Aiming to consider the energy efficiency as one important criterion in the decision-making process, energy-related parameters should be identified and used. Note that since different devices spent different amounts of energy it is possible to choose the device that has lower energy consumption. Potential energy related parameters are:

- Energy consumed during the execution of an operation, which reflects the energy consumption by a device to perform an operation (for example a workstation making a drill or a conveyor transferring a pallet). Normally, it is calculated by multiplying the energy consumption per minute by the processing time.

- Energy consumed during transportation, which reflects the energy consumed during the transportation of materials by the shop floor devices. In production environments it is important to reduce the movements of materials but above all it is necessary to reduce the energy consumption associated to the transportation operation.

- Energy consumed for the set-up, which reflects the amount of energy that it is necessary to setup a device. Combining with the setup time it is possible to power off or stand by the devices when they are not used and only setup the device when they are really needed.

The determination of the energy consumption for each device requires the monitoring of each one, for example using proper software packages available on the market. These gathered values are registered over the time, and statistically treated to get more reliable values. After the statistical treatment they can be used by the decision support system as a decision criterion. The application of learning methods can be useful to predict new patterns based on the device historical data.

\section{EXPERIMENTAL CASE StUdy}

An experimental case study is used to illustrate how the decision-making system, based on the knowledge extracted 
from the Petri nets models, for service-oriented systems works in practice, considering amongst others, the minimization of the energy consumption.

\section{A. Description of the Case Study}

The case study corresponds to the FlexLink ${ }^{\circledR}$ Dynamic Assembly System (DAS) 30, comprising several unidirectional and cross conveyors arranged in a closed-loop configuration, and two lifters connecting the upper and lower systems, as depictured in Fig. 2.

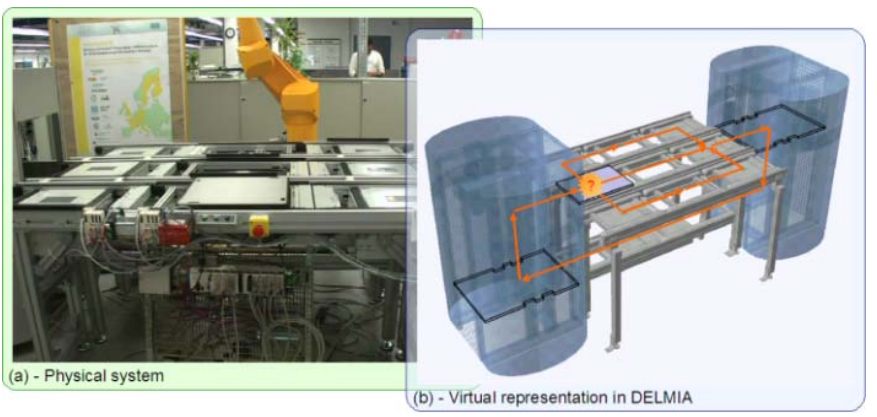

Fig.2. Physical and virtual representation of the case study scenario.

When circulating in the system, a pallet is faced with several decision points, as the one illustrated in the figure. The decision point represents a fork in the paths of the workpiece, upon which it can either continue straight on to the end lifter, or turn in the direction of one of the two workstations. The decision to be taken at this point is related to decide which workstation should perform the operation and consequently which the path to be chosen.

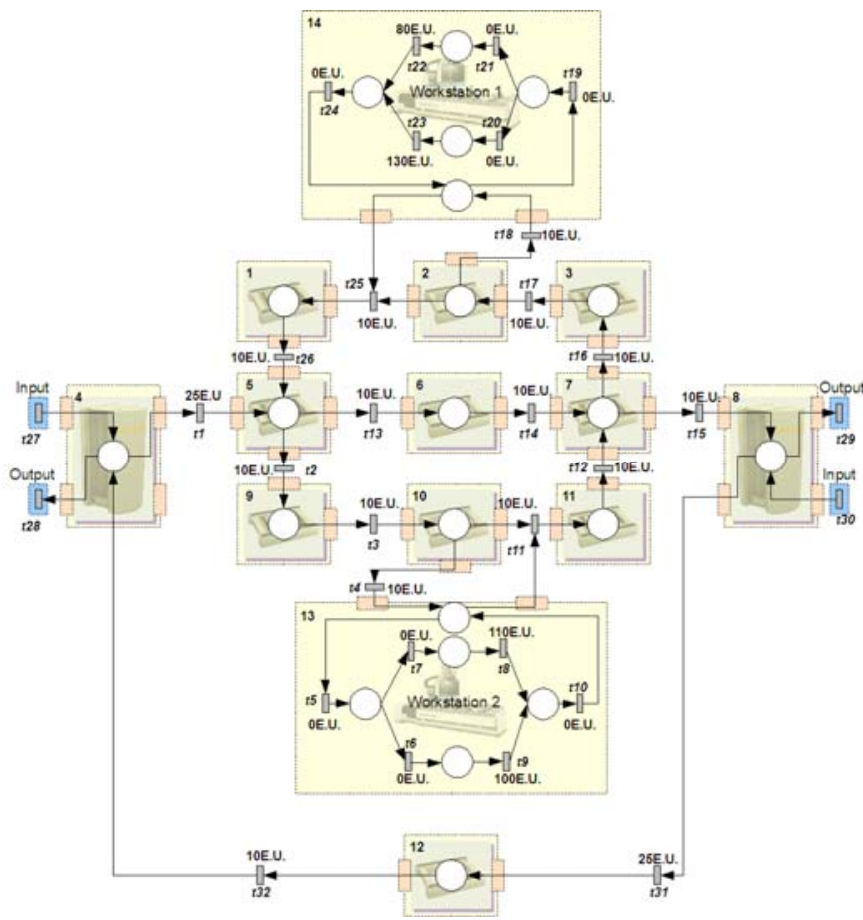

Fig.3. High-level Petri net model for the case study.

The control of the system is defined in a High-Level Petri nets model, illustrated in Fig. 3, which shows the global behavior in the different operation modes, exposed as services.

In the example, there are two workstations (represented by the transitions $t_{9}$ and $t_{23}$ ) with similar operations but with different energy consumption indexes. The knowledge extracted from the structure of the High-level Petri nets model of Fig. 3 is the incidence matrix and the set of $\mathrm{T}$ invariants. This Petri nets model has several T-invariants, but combining them with the workplan, only 3 are valid to execute the expected operation (i.e. in the workstation $\# 1$ or \#2). They have the following physical meaning:

$-x_{1}=\left\{t_{2}, t_{3}, t_{4}, t_{5}, t_{6}, t_{9}, t_{10}, \ldots\right\}$, representing the work cycle that conveys the pallet to the right and contains the workstation \#2 (i.e. the transition $t_{9}$ ).

$-x_{2}=\left\{\mathrm{t}_{13}, \mathrm{t}_{14}, \mathrm{t}_{16}, \mathrm{t}_{17}, \mathrm{t}_{18}, \mathrm{t}_{19}, \mathrm{t}_{20}, \mathrm{t}_{23}, \mathrm{t}_{24}, \ldots\right\}$, representing the work cycle that conveys the pallet forward and contains the workstation \#1 (i.e. the transition $t_{23}$ ).

$-x_{3}=\left\{\mathrm{t}_{2}, \mathrm{t}_{3}, \mathrm{t}_{11}, \mathrm{t}_{12}, \mathrm{t}_{16}, \mathrm{t}_{17}, \mathrm{t}_{18}, \mathrm{t}_{19}, \mathrm{t}_{20}, \mathrm{t}_{23}, \mathrm{t}_{24}, \ldots\right\}$, representing the work cycle that conveys the pallet to the right and contains the workstation \#1 (i.e. the transition $\left.t_{23}\right)$.

Having more than one valid alternative to perform the operation, the decision support system will analyze these $3 \mathrm{~T}$ invariants according to a set of decision criteria.

\section{B. Analysis the Decision-Making System using Energy Criteria}

The decision procedure evaluates the set of T-invariants (i.e. the service invariants) according to pre-defined decision criteria, e.g. productivity, resource utilization or energy.

The first scenario considers only one criterion that is the processing time parameter. The objective in this decision process is to find the alternative that provides faster processing. The processing times assumed in this scenario are 2 t.u. for transportation operations, 80 t.u. for the workstation $\# 1$ and 130 t.u. for the workstation \#2.

Applying the decision function proposed in the previous section, and considering only the time processing criterion, the achieved results are,

$$
\mathrm{f}_{1 \text { st-scenario }}(\mathrm{i})=(136,90,94)
$$

From the previous analysis it is clear that the better solution to execute the desired operation, and taking into consideration only the time processing criterion, is the one specified by the T-invariant $x_{2}$, i.e. conveying the pallet forward and using the workstation $\# 1$. In spite of the longer path to achieve the workstation, for example when compared with the path to achieve workstation \#2, this is sustained due to the lower value of processing time of workstation \#1.

A second scenario considers the energy consumption and the processing time parameters, with equal criteria weight. Here, the decision will be taken considering the need to achieve fast processing and also regarding the energy consumption. The values of energy consumed by the resources to execute the operations are represented in Fig. 3. Namely, transfer operations performed by conveyor devices spent 10 e.u. per operation (see for example the transitions $t_{2}$ 
and $t_{3}$ ), and the processing operations performed by workstations spent more energy (100 and 110 e.u. for workstation \#2 and 80 and 130 e.u. for workstation \#1).

Applying the proposed evaluation function, the achieved results are,

$$
\mathrm{f}_{\text {2nd-scenario }}(\mathrm{i})=(266,270,294)
$$

Now, the selected solution is given by the T-invariant $x_{1}$ that conveys the pallet to the right and uses the workstation $\# 2$. The achieved result shows that in spite of the workstation \#1 has shorter processing time, the best solution is the one that considers a longer path and the workstation \#2, since it presents even higher energy saving (due to the energy saving of the workstation \#2 and the elements of the path). This illustrates that the proposed approach improves the system energy efficiency when compared with non-energy-aware systems. In fact, if we only consider productive parameters, as usual production systems do, the decision is one but considering energetic parameters the decision is another.

The experimental results show the flexibility of the decision support system, namely due to the knowledge extracted from the Petri nets behavior model and to the parameterization of the decision criteria that allows adjusting dynamically the decision-making. Note that the parameterization of the decision criteria is dependent of the system objectives but also strongly dependent of the learning mechanisms embodied in the decision support system.

These very simple scenarios, applied to the case study, can be easily improved and extended to big systems.

\section{CONCLUSIONS AND FUTURE WORK}

The work presented in this paper is focused in the development of modular, distributed and green process control systems, devoting a special attention to the energy efficiency in manufacturing environments, which are traditionally characterized by energy-intensive consumption. Newer approaches contributing for a green and saving environment (i.e. a better and efficient use of energy, a reduction of emissions and a greater energy independence), require the adoption of more energy-efficient technologies and the introduction of control systems that better manage the energy efficiency criteria.

For this purpose, an innovative approach for the decision support systems is introduced, which considers the knowledge extracted from the Petri nets models used to describe and execute the control processes in service-oriented automation systems. The decision-making is based on the application of a set of criteria, namely the traditional productive ones combined with some energy related parameters, such as the energy consumption. The parameterized criteria vector is applied to the T-invariants extracted from the Petri nets behavior model, allowing achieving the better solution from the alternatives ones.

The proposed approach was illustrated using a case study comprising a real assembly system. It was also discussed how traditional non-energy aware control systems handle the same scenario being highlighted the benefits of the proposed approach. As future work, a further analysis on energy related criteria should be performed and the decision mechanism should be enhanced to handle the workplan as a whole, becoming a non-myopic decision system.

\section{ACKNOWLEDGMENT}

The authors would like to thank the European Commission and the partners of the EU IST FP6 project "Service-Oriented Cross-layer infrastructure for Distributed smart Embedded devices" (SOCRADES), and the European ICT FP7 project "Cooperating Objects Network of Excellence" (CONET) for their support.

\section{REFERENCES}

[1] F. Jammes and H. Smit, "Service-oriented Paradigms in Industrial Automation", IEEE Transactions on Industrial Informatics, vol. 1, n. 1, pp. 62-70, 2005.

[2] M. Bichier and K.-J. Lin, "Service-oriented Computing", IEEE Computer, vol. 39, n. 3, pp. 99- 101, 2006

[3] J. M. Mendes, P. Leitão, A. W. Colombo and F. Restivo, "Serviceoriented Process Control using Modular High-Level Petri Net", Proceedings of the 6th IEEE International Conference on Industrial Informatics, South Korea, pp. 750-755, 2008.

[4] M. Silva and R. Valette, "Petri nets and flexible manufacturing", In Advances in Petri nets, LNCS 424, Springer-Verlag London, pp. 374417, 1990.

[5] T. Murata, "Petri nets: Properties, analysis and applications", IEEE, vol. 77, pp. 541-580, April 1989.

[6] M. Silva, "Las Redes de Petri en la Automática y la Informática, Editorial AC, 1985.

[7] K. Feldmann and A.W. Colombo, "Material Flow and Control Sequence Specification of Flexible Production Systems using Coloured Petri Nets", International Journal of Advanced Manufacturing Technology, Springer London, pp. 760-774 1998.

[8] K. Feldmann and A.W. Colombo, "Monitoring of Flexible Production Systems Using High-Level Petri Nets", Control Engineering Practice, n. 7, pp. 1449-1466, 1999.

[9] M. Combacau and M. Courvoisier, "Process Failures Diagnosis in F.M.S. Real-time Control: an Approach Combining Rule-based Systems and Petri nets", In Proceedings of IEEE International Conference of Robotics and Automation, pp. 174-180, 1991.

[10] R. Du, M.A.Elbestavi and S. Wu, "Automated monitoring of Manufacturing processes, Part 1: monitoring methods", Journal of Engineering for Industry, vol. 117, pp. 121-130, 1995.

[11] E. Kreyszig, "Introductory Functional Analysis with Applications", Wiley, 1989.

[12] G. Memmi and G. Roucairol, "Linear Algebra in Net Theory", In LNCS 84, Springer Verlag N.Y., pp. 213-223,1980.

[13] A. Giua and F. DiCesare, "Petri net Structural Analysis for Supervisory Control", IEEE Transactions on Robotics and Automation, vol. 10, n. 2, pp. 185-195, 1994.

[14] J.M. Couvreur and J. Martinez, "Linear Invariants in Commutative High-Level Nets", In LNCS 483, Springer Verlag London , pp. 146165, 1991.

[15] P. Leitão, J. M. Mendes and A. W. Colombo, "Decision Support System in a Service-oriented Control Architecture for Industrial Automation", In Proc. of the IEEE Int'l Conference on Emerging Technologies and Factory Automation, pp. 1228-1235, 2008.

[16] J. Martinez and M. Silva, "A Simple and Fast Algorithm to Obtain All Invariants of a Generalized Petri net", In C. Girault and W. Reisig (eds.) Fachberichte Informatik, vol.52, Springer Verlag, pp. 301-310, 1982. 